\title{
Téoros
}

Revue de recherche en tourisme

\section{Les mesures sociales pour accroître le départ en vacances et}

l'accès au tourisme

\section{Une relance est-elle possible?}

\section{Louis Jolin}

Volume 22, numéro 3, automne 2003

L’accessibilité, une conquête inachevée

URI : https://id.erudit.org/iderudit/1071341ar

DOI : https://doi.org/10.7202/1071341ar

Aller au sommaire du numéro

Éditeur(s)

Université du Québec à Montréal

ISSN

0712-8657 (imprimé)

1923-2705 (numérique)

Découvrir la revue

Citer cet article

Jolin, L. (2003). Les mesures sociales pour accroître le départ en vacances et l'accès au tourisme : une relance est-elle possible ? Téoros, 22(3), 10-15.

https://doi.org/10.7202/1071341ar d'utilisation que vous pouvez consulter en ligne.

https://apropos.erudit.org/fr/usagers/politique-dutilisation/ 


\section{Les mesures sociales pour accroître le départ en vacances et I'accès au tourisme}

\section{Une relance estelle possible?}

\section{Louis Jolin}

D epuis l'avènement des congés payés, plusieurs pays ont cru bon d'adopter des mesures sociales, voire des politiques sociales, pour rendre les vacances et le tourisme accessibles au plus grand nombre. S'il est vrai que, à la fin de la Deuxième Guerre mondiale, l'immense marché créé par l'octroi de congés annuels payés dans plusieurs pays industrialisés a obtenu satisfaction grâce à une offre commerciale appropriée - l'essor du tourisme de masse ne fait plus aucun doute ! -, il n'empêche que plusieurs catégories de population ne peuvent partir en vacances sans une intervention des pouvoirs publics. Encore de nos jours, malgré les mesures sociales, la moitié - et même plus - de la population dans nombre de pays industrialisés ne peut partir en vacances, notamment, et surtout, pour des raisons socioéconomiques, sans compter les difficultés d'accès aux installations pour les personnes à capacité physique restreinte. Même en France, pays qui accorde légalement cinq semaines de vacances et qui est reconnu pour ses politiques sociales, environ $40 \%$ des Français ne partent pas en vacances ; au Québec, plus de $50 \%$ des Québécois ne voyagent pas (voyage de plus d'une journée en dehors de leur domicile habituel) pendant leurs vacances (période de quatre jours ou plus, autres que les fins de semaine $)^{1}$.

La mise en place de mesures sociales ne va pas de soi. Ces mesures sont le résultat de luttes dirigées dans plusieurs pays par des syndicats et des associations de citoyens, qui ont généralement été menées sous l'étendard du droit aux congés et du droit aux loisirs, tels que reconnus dans certaines déclarations internationales des droits ${ }^{2}$, mais aussi avec des arguments de nature économique (élargissement du marché, retombées économiques, etc.).

Quelles sont ces mesures ? Sont-elles constitutives d'une réelle politique sociale ? S'inscrivent-elles dans une politique sociale élargie qui vise l'inclusion et la cohésion sociales ou la promotion de la solidarité ? Comment les évaluer ? Ces questions, auxquelles nous ne répondrons que partiellement, constituent l'ossature du présent article.

\section{Un inventaire de mesures sociales}

Le Bureau international du tourisme social (BITS) a entrepris ces dernières années d'inventorier les principales mesures sociales mises en place par les pouvoirs publics et encore effectives dans les pays de l'Europe de l'Ouest surtout (le berceau du tourisme social mondial), mais aussi dans quelques autres pays, notamment dans les Amériques. Cette démarche a donné lieu à la publication de quelques documents .

Les auteurs des documents du BITS ont classé les mesures selon diverses typologies. Tout d'abord, ils ont proposé un classement selon la nature de l'intervention et les acteurs concernés. Trois types d'intervention ont ainsi été retenus :

1. les pays dont l'intervention de la collectivité ou de l'État est directe (à titre d'exemples, la Belgique, la France, le Portugal, le Mexique) ;
2. les pays dont l'intervention est surtout réalisée sur la base d'un partenariat entre acteurs sociaux (la Suisse, l'Autriche, le Danemark, la Suède, la Colombie, le Brésil...) ;

3. les pays dont l'intervention est de nature caritative, ce qui signifie que l'État joue un rôle réduit (le Royaume-Uni, l'Irlande, l'Allemagne sont des pays représentatifs de cette approche).

Une autre typologie permet de distinguer les types d'aides ou de subventions. Les documents du BITS font état de deux types d'aides :

1. l'aide « à la pierre » qui concerne toutes les aides destinées aux investissements (construction de centres de vacances, rénovation, réhabilitation, entretien et mise aux normes);

2. l'aide à la personne qui concerne directement les individus (enfants pour les centres de vacances collectives, familles pour les vacances familiales).

À ces deux types d'aides, nous en ajoutons un troisième que nous pourrions appeler l'aide au fonctionnement des associations qui gèrent les établissements ou des programmes de voyages et de séjours de vacances.

À titre d'exemples et sans vouloir être exhaustif, on trouve en Belgique des programmes de subventions pour la création et l'amélioration d'établissements de vacances pour les jeunes et les familles surtout, pouvant couvrir jusqu'à $75 \%$ du coût total mais, depuis une vingtaine d'années, les interventions de l'État visent en priorité la modernisation et l'adaptation des structures d'accueil exis- 
tantes plutôt que le développement de nouveaux établissements. L'intervention de l'État central diminue à la faveur d'une décentralisation vers les trois grandes régions (flamande, française et allemande). L'aide à la personne en Belgique prend principalement la forme d'un système d'aide au départ en vacances, appelé le pécule ou le double pécule de vacances : le montant de l'aide, calculé sur la base du salaire, correspond dans le cas de vacances complètes ( 24 jours) à un salaire double de trois semaines et trois jours. Ce pécule correspond à 14,75\% du salaire brut. Le système mobilise les employeurs, l'Office national de sécurité sociale et l'Office national des vacances annuelles (OVNA). En outre, l'État et les administrations fournissent de l'aide aux associations pour le développement et la promotion de leurs actions.

En France, même si $90 \%$ de l'aide à la pierre a été effectuée avant 1986 grâce aux crédits de l'État, de la Caisse nationale des allocations familiales et des comités d'entreprises, il reste encore des programmes d'aide pour la modernisation et la rénovation des centres de vacances du tourisme social (par le biais du Plan Patrimoine, de l'intervention récente en ce domaine des collectivités locales et de l'Agence nationale pour le chèque-vacances - ANCV - pour des aides complémentaires). L'aide à la personne est particulièrement développée dans ce pays : outre la politique du treizième mois (au minimum), on trouve le programme des bons-vacances des Caisses d'allocations familiales, dont la mission prioritaire est d'aider les jeunes et les enfants à partir en vacances; les chèques-vacances émis par l'ANCF depuis 1981, qui s'adressent aux salariés, mais qui permettent de constituer un fonds d'investissement pour des aides complémentaires à la pierre et des bourses pour les plus démunis ; la Bourse Solidarité Vacances, de création récente (1999) dans le cadre de la mise en œuvre de la Loi sur l'exclusion, dont l'objectif est, notamment, de rassembler des offres de vacances à très bas prix pour permettre à des familles en situation difficile de partir en vacances. Outre ces dispositifs, il faut compter en France sur l'action des comités d'entreprises et sur divers autres programmes du Secrétariat au tourisme, en concertation avec les associations de tourisme, pour promouvoir le tourisme des jeunes, le tourisme des aînés et celui des personnes handicapées.

Évidemment, le cadre limité du présent article ne nous permet pas d'exposer en détail l'ensemble des mesures des divers pays, mais rappelons quelques autres exemples significatifs :

- La Grèce a mis sur pied, sous la responsabilité de l'Office national du tourisme grec, un programme d'aide à la personne qui consiste à fournir des carnetsvacances nominatifs, réservés aux personnes à faibles revenus; les aides couvrent plus ou moins $40 \%$ du coût du séjour qui ne peut excéder une semaine.

- En Italie, même si l'on ne peut faire état d'investissements importants de la part des pouvoirs publics pour réaliser un réseau réceptif et des services du tourisme social, on peut souligner quelques aides à la pierre sur le plan régional et un appui aux activités touristiques proposées par les associations sans but lucratif, culturelles, sociales, religieuses.

- Au Portugal, les travailleurs ont aussi droit au treizième mois et les « seniors » bénéficient d'un important programme d'aide au départ en vacances qui encourage l'exploitation des équipements touristiques en basse saison. Dans ce pays, le tourisme social s'articule principalement autour de l'Institut national pour la promotion des temps libres des travailleurs (INATEL) qui gère treize centres de vacances et trois campings.

- La Suisse est le berceau de la formule du chèque-vacances : la Caisse REKAqui émet toujours les chèques-vacances depuis près de cinquante ans est, par contre, une coopérative et non une institution publique; l'État intervient indirectement par le biais de la défiscalisation de la contribution des employeurs.

- Le Danemark est surtout connu pour avoir mis sur pied le Fonds de vacances du marché du travail (Arbejdmarketes Ferie Fond) qui a pour mission de recueillir, à chaque trimestre, auprès des entreprises les montants destinés aux paies de vacances $(12,5 \%$ du salaire des travailleurs) et d'investir cet argent pendant un certain temps dans les produits financiers de l'État avant de les remettre aux travailleurs lors de leurs vacances : la rémunération du capital investi constitue la partie essentielle des ressources du Fond qui appuie ainsi le développement des infrastructures de vacances et le départ en vacances.

- L'Institut mexicain de sécurité sociale est propriétaire et gestionnaire d'un réseau de quatre centres de vacances de grande envergure destinés à l'accueil des Mexicains de la classe moyenne.

- La Colombie a un système d'allocations familiales qui repose sur trois piliers : la Super-Intendance des allocations familiales (organisme public qui dépend du ministère du Travail), les Caisses de compensation (organismes privés à but non lucratif) et les allocations comme telles provenant des cotisations des employeurs à hauteur de $4 \%$ du salaire des travailleurs ; une partie des allocations est versée sous forme de services touristiques (établissements de vacances, programmes de voyages, etc.).

- Le Royaume-Uni possède très peu de centres de vacances gérés par des collectivités, des syndicats ou des associations en comparaison avec d'autres pays européens et compte sur le rôle des associations dans une perspective caritative ; il existe néanmoins quelques programmes d'aides pour les enfants dans le besoin, en vertu du Children's Act ou pour permettre des congés de « répit » destinés aux personnes qui s'occupent à plein temps d'un membre de la famille, handicapé ou malade, ou destinés aux familles d'accueil. Récemment, le gouvernement britannique a lancé une campagne Tomorrow Tourism pour encadrer les politiques liées au tourisme et l'accessibilité des pratiques touristiques et culturelles est apparue comme une préoccupation.

- Enfin, le Québec a mis sur pied, au cours des trente dernières années, des programmes de financement visant à favoriser le départ en vacances et l'accessibilité au tourisme, principalement 
celui consacré aux associations nationales de loisir touristique et de plein air (aide au fonctionnement et aux projets) et celui qui concerne les établissements de vacances (les camps de vacances et les camps familiaux, car l'État s'est retiré officiellement du financement des bases de plein air et des auberges de jeunesse, bien qu'il intervienne encore à l'occasion à la faveur de programmes généraux liés aux infrastructures ou à l'économie sociale). Quelques mesures, notamment l'appui à Kéroul ${ }^{4}$, visent à accroître l'accessibilité aux personnes à capacité physique restreinte.

Ce bref survol n'a d'autres prétentions que d'illustrer, sur la base des travaux du BITS, ce que l'on peut entendre par mesures sociales pour le départ en vacances et l'accessibilité au tourisme. Il révèle la diversité des actions, des acteurs concernés et des clientèles visées. Il oublie cependant diverses mesures qui ont des incidences sur l'accessibilité au tourisme sans nécessairement posséder cette finalité première (réductions sur les transports en commun, tarifs réduits dans les musées pour certaines clientèles, développement de réseaux de parcs nationaux et régionaux, de pistes cyclables, de sentiers de randonnée, etc.). Ce survol ne rend pas suffisamment compte de l'évolution des mesures dans une période où l'État-Providence est menacé de toutes parts et ne met pas en perspective leurs interactions au sein d'un même pays, ni leur intégration au sein de véritables politiques destinées à réduire la pauvreté ou visant l'inclusion et la cohésion sociales.

\section{Peut-on réellement parler de politiques sociales ?}

On peut définir une politique gouvernementale comme un ensemble d'actions concertées, engagées par un gouvernement dans le but d'atteindre des résultats déterminés. Peu de gouvernements (à l'exception de ceux de la Belgique et de la France...) ont adopté de véritables politiques sociales du tourisme avec des objectifs clairement affirmés et des actions conséquentes. D'ailleurs, un inventaire des politiques sociales ne retient généralement pas le domaine du tourisme parmi les thèmes principaux. Par exemple, si l'on consulte sur Internet le très beau site consacré à l'évolution des politiques sociales dans le cadre de la mondialisation - [www.politiquessociales.net] animé par le Centre de recherche sur les politiques et le développement social (Université de Montréal), ni le loisir, ni le tourisme ne se trouvent dans la liste des thèmes. On y trouve des rubriques sur les politiques du travail et de l'emploi, sur les questions de licenciement, de délocalisation, de temps de travail, de lutte contre la pauvreté, de sécurité du revenu, de minima sociaux, d'économie sociale et de microcrédit, de cohésion sociale... Le thème du temps de travail est peut-être celui qui semble a priori avoir le plus d'affinités avec celui du loisir, du moins sous l'angle du temps libre et des congés payés.

Les chercheurs du Centre de recherche sur les politiques et le développement social constatent par ailleurs une stagnation, voire une réduction des politiques sociales dans les pays industrialisés, ce qui ne contribue certes pas à leur élargissement à de nouvelles thématiques clairement affichées.

En politique intérieure, on a constaté au cours des deux dernières décennies, en certains pays des reculs de la couverture sociale, ailleurs un certain immobilisme sur la question, plus globalement une redéfinition des principes d'universalité et du concept de droits sociaux pour se concentrer sur une aide ciblée et conditionnelles.

Cette évolution correspond à ce que nous avons pu constater dans notre analyse des mesures sociales qui touchent le tourisme, la plus importante étant la diminution importante des programmes d'aide à la pierre et leur remplacement, du moins partiel, par des programmes d'aide à la personne davantage ciblés. La décentralisation des aides publiques qui oblige une négociation serrée avec les collectivités locales contribue aussi à un éloignement du principe d'universalité. Il en est de même des nouvelles politiques de tarification sur des espaces autrefois gratuits (notamment les parcs provinciaux au Québec).
Dans un tel contexte de redéfinition, de stagnation, voire de réduction des mesures sociales en tourisme, comment alors leur redonner un deuxième souffle ? Peut-on croire à leur affirmation dans le cadre de politiques sociales du loisir et du tourisme autonomes ? L'approche la plus prometteuse nous parait être leur insertion de manière forte et articulée au sein de politiques plus générales portant sur l'inclusion sociale, la cohésion sociale, la solidarité...

La décentralisation dans le secteur du tourisme, la mise en œuvre de partenariats organiques publics-privés, particulièrement sur le plan de la promotion et de la commercialisation, la délégation d'un certain nombre de mandats au secteur privé - autrefois assumés par l'État - ne favorisent pas l'intégration d'une approche sociale dans la politique touristique ellemême. Il faut changer de perspectives et de stratégies.

Dans un monde d'insécurité, face à la complexité des problèmes sociaux, malgré l'idéologie néo-libérale qui imprègne nombre des décisions gouvernementales des deux dernières décennies, plusieurs États sont néanmoins préoccupés par les effets de l'exclusion, par l'émergence d'une société duale. Les pays de l'Union européenne sont d'ailleurs invités à élaborer et à rendre publics des plans nationaux d'inclusion sociale. La récente cuvée couvre les années 2003-2005. Quelles est la place du loisir et du tourisme comme outils d'inclusion sociale?

\section{Les plans nationaux d'action pour l'inclusion sociale des pays de l'Union européenne}

Une brève analyse des plans (Commission européenne, 2003) de quinze pays (le Portugal n'ayant pas encore déposé son plan pour 2003-2005 au moment de notre recherche, nous avons considéré le plan 2001-2003) induit quelques conclusions préliminaires.

Six des quinze pays étudiés n'ont pas inclus de mesures ni de politiques concernant le tourisme, les sports ou le loisir comme moyens de lutter contre la pauvre- 


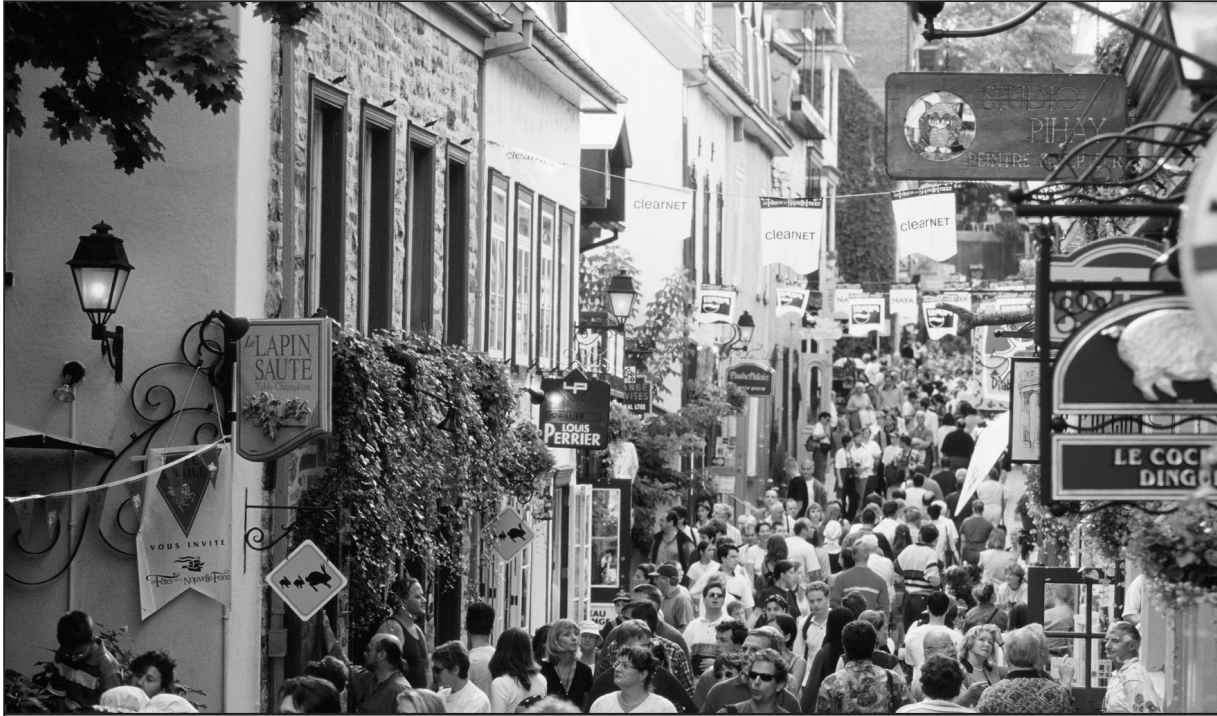

L'accessibilité et le tourisme de masse ne vont pas toujours de pair.

Photo : Linda Turgeon / Tourisme Québec.

té et de favoriser l'inclusion sociale ; ces pays sont : l'Autriche, les Pays-Bas, l'Irlande, l'Allemagne, la Finlande et l'Espagne. Quelques nuances s'imposent pourtant : en Allemagne et en Finlande, en matière de bonnes pratiques, on trouve des projets et des réalisations originales visant l'amélioration de l'aménagement urbain et des loisirs pour les enfants (en Allemagne, le projet $\mathrm{Mo.Ki}$ Monheim for Children; en Finlande, le modèle Tuku de promotion du bien-être des enfants et des jeunes).

Les pays qui considèrent le tourisme, la culture, le sport et les loisirs comme facteurs d'inclusion sociale et de lutte contre la pauvreté sont la France, la Belgique, la Grèce, le Luxembourg, l'Italie, la Grande-Bretagne, le Danemark, la Suède et le Portugal (pour ce dernier, dans son plan 2001-2003). De ces pays, seuls la France, la Belgique, la Grèce, l'Italie et le Portugal mentionnent explicitement le tourisme. Les visions et les approches diffèrent cependant d'un pays à l'autre.

L'Italie mise sur l'échange des étudiants entre les pays comme un moyen de connaître l'Autre et de s'ouvrir au monde extérieur, le tourisme y étant mentionné comme favorisant la formation informelle des jeunes.
La France, avec des politiques de tourisme social traditionnellement fortes, l'a évidemment intégré dans son plan. Elle mentionne explicitement le programme de bourses de solidarité-vacances tout comme le programme « ville-vie-vacances » qui permet à près de 800000 jeunes qui ne partent pas en vacances de bénéficier de nombreux projets à caractère sportif et culturel. La France, comme le Luxembourg qui salue l'initiative d'une $\mathrm{ONG}^{6}$ en faveur des handicapés sensoriels, propose des projets de tourisme pour les personnes handicapées et prévoit une amplification de son programme « Tourisme et Handicap ».

Le programme le plus exhaustif en matière de tourisme et de loisir est présenté par la Belgique : centres de vacances, promotion du sport pour tous, subventions des centres sportifs locaux, diverses initiatives pour soutenir les vacances en groupes et les vacances individuelles des personnes à faibles revenus et de leurs enfants (dans les communautés francophone, flamande et germanophone), émission de chèques-culture pour l'accès gratuit à des spectacles ou à des musées...

La Grèce se distingue aussi par des mesures qui révèlent que l'exclusion sociale n'est pas uniquement un problème économique, ce qui résulte en une approche très globale qui comprend la culture et le tourisme : on propose des excursions gratuites de courte durée, des billets de théâtre et des livres gratuits aux populations rurales, ainsi que la création de bibliothèques mobiles.

La Grèce et le Danemark prévoient des activités sportives comme moyens d'intégration des immigrants à la société d'accueil. Dans son programme d'intégration des gitans, le ministère de la Culture grecque vise à promouvoir l'héritage culturel des gitans ainsi qu'à leur présenter la culture grecque.

Le Portugal est le seul pays qui présente le tourisme social des aînés comme un moyen de leur assurer une qualité de vie. Les ânés sont aussi mentionnés dans le plan belge comme bénéficiaires potentiels d'autres formes de loisir et de sports.

Au Québec, soulignons que l'Assemblée nationale a adopté, en décembre 2002, une loi visant à lutter contre la pauvreté et l'exclusion sociale et doit rendre public d'ici peu un plan d'actions. Quelle place y occuperont le tourisme et les loisirs ? Ferat-on référence aux programmes mis en place par le ministère des Relations avec le citoyen et de l'Immigration, comme celui qui vise la francisation des nouveaux arrivants et qui comporte des activités de loisir et des excursions (visites de la capitale nationale, visites de musées, excursions aux pommes, à la cabane à sucre...) ?

Que conclure de ces quelques exemples ? Que certains États sont disposés à adopter des mesures sociales du tourisme dans leur politique d'inclusion sociale, que d'autres ne le font pas ou n'y ont tout simplement pas pensé, mais, plus globalement, que la pression des divers groupes sociaux et des associations de tourisme social pourrait s'orienter dans cette voie prometteuse. Encore que le concept d'inclusion sociale est peut-être réducteur, trop lié à l'objectif de diminution de la pauvreté - ce qui n'est pas un mal en soi, au contraire -, mais d'autres concepts comme celui de la cohésion sociale ou celui de la solidarité sont aussi porteurs de politiques et de stratégies dans une perspective encore plus ouverte. 


\section{D'autres concepts porteurs}

La cohésion sociale est un concept-phare qui rappelle constamment le besoin d'être collectivement attentifs et sensibles à toute forme de discrimination, inégalité, marginalité ou exclusion. La cohésion sociale n'est pas un concept homogénéisateur, basé uniquement sur des formes traditionnelles d'intégration sociale, qui pourtant sont importantes, telles que l'identité, le partage de la même culture, l'adhésion aux mêmes valeurs ${ }^{7}$.

Plusieurs chercheurs en travail social, en science politique (dont le groupe Naxus) s'y réfèrent comme un concept pouvant servir de socle à de nouvelles politiques sociales.

Le concept de solidarité, dont le sens commun réfère à la dépendance mutuelle entre les hommes, et au sentiment qui les pousse à s'accorer une aide mutuelle, a une longue histoire qui remonte à la Révolution française dans sa consolidation normative. Il est intimement lié aux concepts et aux pratiques de justice sociale, d'engagement social, d'universalité telle que définie par Habermas comme "l'égal respect pour chacun et de la solidarité avec tout ce qui porte visage humain » (1992: 108), de nouvelle démocratie réclamée par nombre de mouvements sociaux dénonçant la surexploitaion des ressources naturelles, l'inégalité entre les sexes, le racisme, l'exclusion, etc... (Melkevik, 2000 : 17-33).

Ce concept de solidarité a donné lieu, dans certains pays, à des politiques et des législations de « solidarité ». C'est un terreau fertile pour l'éclosion de nouvelles mesures d'accessibilité aux vacances et au tourisme non seulement pour les divers segments de population qui souhaitent partir en vacances, mais aussi pour les communautés locales qui accueillent les vacanciers et les touristes. L'accessibilité au tourisme ne concerne pas seulement les visiteurs potentiels, mais aussi les visités qui doivent avoir accès aussi bien à leurs ressources touristiques qu'aux bénéfices du tourisme... tout en voyant à ne pas dilapider les ressources.
La solidarité s'entend également sur les plans national et international. Face à la mondialisation néo-libérale, on trouve des réalisations de la société civile qui démontrent qu'il y a d'autres façons de voir la mondialisation. En ce sens, le tourisme social, autant par les mesures et les politiques sociales qu'il induit que par les initiatives et les réalisations des associations, est un protagoniste de tout premier plan de l'économie sociale et solidaire. Sur le plan national, il « est la démonstration que pertinence économique et gestion sont compatibles avec accessibilité au plus grand nombre, mixité sociale et développement local. Le tourisme en économie sociale est aussi l'illustration que valeur ajoutée économique peut rimer avec valeur ajoutée sociale »(Chauvin, 2002 : 4 e couvert.). Sur le plan international, le tourisme s'inscrit dans une mouvance de globalisation des solidarités à l'échelle de la planète au sein de laquelle le social reste au poste de commande, l'économie devient un instrument et non une fin en soi et la protection de l'environnement «une conditionnalité nouvelle dans les choix économiques qui s'opèrent » (Groupe d'économie solidaire du Québec, 2001 : 7).

\section{Un programme de recherche}

Ces quelques réflexions ont un double but : orienter l'action des intervenants et proposer un plan de recherche. En ce qui concerne l'action, de nouvelles mesures sociales, voire de réelles politiques sociales, verront le jour sur le terrain de l'accessibilité aux vacances et au tourisme si l'on réussit l'arrimage entre ces objectifs et ceux plus globaux que représentent l'inclusion sociale, la cohésion sociale ou la solidarité. Ce type d'arrimage pourrait aussi s'opérer, selon la conjoncture, avec des objectifs de santé physique et mentale et d'éducation des citoyens, principalement dans une perspective de prévention et d'éducation informelle et permanente.

La recherche sur les mesures et les politiques sociales dans le domaine des vacances et du tourisme reste embryonnaire. Les travaux du BITS ont un caractère surtout descriptif. Quelques auteurs comme le professeur Jean Froidure
(1997) ont su tracer les évolutions et les transformations récentes dans un contexte national (France), mais il n'y a pratiquement pas de travaux d'évaluation pour mesurer l'efficacité ou l'efficience des mesures ou des politiques sociales. Un programme de recherche pourrait s'articuler autour de trois axes :

- l'évaluation des mesures et des politiques sociales existantes selon un certain nombre de critères (efficacité, efficience, mais aussi niveau d'engagement de la société civile, etc.) ;

- une étude du degré de leur arrimage avec des politiques sociales élargies (inclusion sociale, solidarité, santé, etc.) ;

- une réelle analyse comparative des mesures sociales sur le plan international en tenant compte du niveau développement et des caractéristiques des pays.

L'inventaire desdites mesures devrait être réalisé de façon permanente et devenir l'objet d'un observatoire spécifiquement dédié ou, préférablement, être intégré comme champ spécifique dans le menu d'un observatoire aux préoccupations plus étendues (sur les politiques sociales, le loisir ou le tourisme...).

Le comité scientifique du Bureau international du tourisme social, en collaboration avec quelques universités et centres de recherche, mais aussi avec de grandes associations nationales $\mathrm{du}$ tourisme social, pourrait prendre la direction du programme de recherche. Un projet d'envergure certes, mais indispensable dans la conjoncture actuelle si l'on souhaite réellement une relance et un développement des politiques sociales du tourisme...

Louis Jolin est juriste et professeur au Département d'études urbaines et touristiques de l'Université du Québec à Montréal et responsable $d u$ Comité scientifique $d u$ Bureau international du tourisme social. Il remercie Mme Katia Iankova, étudiante au doctorat en études urbaines, pour son analyse des plans nationaux d'action pour l'inclusion sociale (2003-2005) des pays de l'Union européenne. 


\section{Notes}

1 Les chiffres pour la France proviennent d'une recherche de l'INSEE de 1996. Voir Matteudi (1997 : 12) ; lors de l'enquête à la grandeur du Québec, en 1995, 58,2\% n'étaient pas partis en vacances dans la dernière année. Voir Stafford et Samson (1996 : 20).

2 Plusieurs luttes ont été menées en vertu de l'article 24 de la Déclaration universelle des droits de l'Homme et des articles du Pacte relatif aux droits économiques, sociaux et culturels, adoptés par l'ONU, reconnaissant à toute personne le droit au repos et aux loisirs et invitant les États à limiter la durée du travail et à obliger l'octroi de congés payés.

3 BITS-Europe (2000), Actes du Séminaire de Strasbourg, Bruxelles, Bureau international du tourisme social ; BITS-Union européenne (2001), Les concepts de tourisme pour tous et de tourisme social dans l'Union européenne, Séminaire de Bruges de juin 2001, Bruxelles, Bureau international du tourisme social ; BITS (2002), « Les politiques sociales » et BITS-Amériques (2002) «L'offre du tourisme social dans le monde », contributions à Étude sur la viabilité du tourisme social au Mexique, réalisée par l'Université autonome de Mexico pour le compte de CESTUR et SECTUR (publiée en espagnol).

4 Organisme québécois à but non lucratif qui informe, représente et fait la promotion du tourisme et de la culture accessibles.

5 Voir le site Internet du Centre de recherche sur les politiques et le développement social de l'Université de Montréal, « Pourquoi ce site ? » [www.politiquessociales.net].

6 Organisation non gouvernementale.

7 Extrait de la Revue canadienne de droit et société, vol.16, $\mathrm{n}^{\circ} 2,2001$. Ce numéro rappelle l'importance des droits sociaux dans la détermination du concept de cohésion sociale.

\section{Bibliographie}

Centre de recherche sur les politiques et le développement social de l'Université de Montréal, site web : www.politiquessociales. net.

Chauvin, Jacques (2002), Le tourisme social et associatif en France. Acteur de l'économie sociale, Paris, L'Harmattan.
Commission européenne (2003), Plans nationaux d'action pour l'inclusion sociale (20032005), Bruxelles, juillet.

Froidure, Jean (1997), Du tourisme social au tourisme associatif, Paris, L'Harmattan, 190 p.

Groupe d'économie solidaire du Québec (2001), "Résister et construire », Une autre mondialisation, Montréal, p. 7.

Habermas, J. (1992), De l'éthique de la discussion, Paris, Cerf.

Matteudi, Guy (1997), Les vacances, facteur d'insertion sociale, Rapport présenté au Conseil national du Tourisme, Paris.

Melkevik, Bjarne (2000), « La solidarité, la philosophie et notre présent », Réflexions sur la philosophie du droit, Québec, Les Presses de l'Université Laval en collaboration avec L'Harmattan, p. 17-33.

Stafford, Jean, et Marcel Samson (1996), Vacances et tourisme 1995, Enquête auprès d'un échantillon de Québécois et de Montréalais sur les comportements de vacances, Québec, Ministère des Affaires municipales.

\section{Nouveau}

\section{Programme court de $1^{\text {er }}$ cycle en gestion du tourisme}

$\rightarrow$ Offert à distance, en ligne et en français

$\rightarrow$ Un programme conjoint de l'ESG-UQAM et de la TÉLUQ

$\rightarrow$ Cinq cours crédités :

- Initiation à la gestion

- Atelier sur les intervenants touristiques

- Gestion des évènements et congrès

- Technologie, distribution et transports

- Environnements administratifs et politiques du tourisme

\section{Pour les conditions d'admission et les modalités d'inscription :}

Service d'accueil et de renseignements 1-888-843-4333 (sans frais partout au Canada) info@teluq.uquebec.ca

www.teluq.uquebec.ca

Inscrivez-vous maintenant !

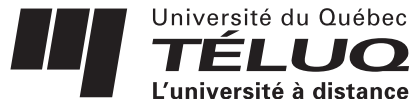

UQĀM ESG

Prenez position 